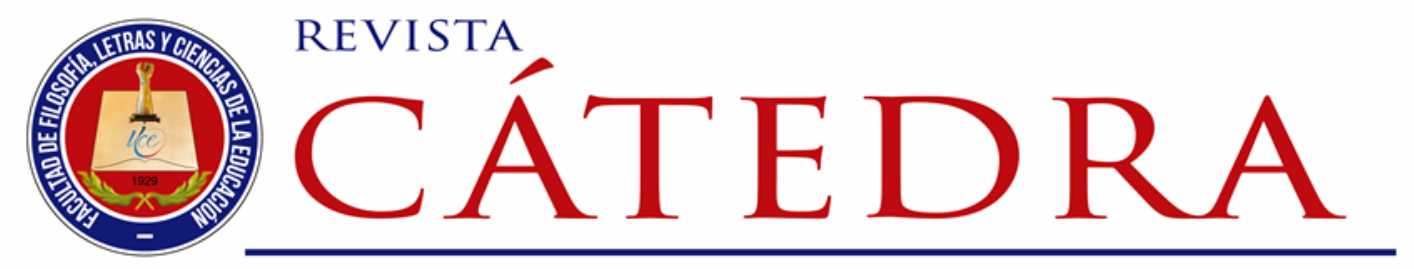

\title{
Asesoría Educativa en el Ecuador: campos de tensión
}

\section{Educational Consulting in Ecuador: Fields of tension}

\author{
Gustavo Vallejo-Villacís \\ Asesoría Pedagógica Consultores \\ asesoria.pedagogica.consultores@gmail.com \\ https://orcid.org/0000-0001-9525-9688
}

(Recibido: 11/06/2020; Aceptado: 15/06/2020; Versión final recibida: 15/08/2020)

Cita del artículo: Vallejo-Villacís, Gustavo. (2020). Asesoría educativa en el Ecuador: campos de tensión. Revista Cátedra, 3(3), 88-110.

\section{Resumen}

La asesoría educativa en el Ecuador emerge en el año 2013 como una función que reemplaza a la supervisión escolar. Prácticamente no existen estudios o análisis sobre esta importante función dentro del sistema organizacional del Ministerio de Educación. El presente artículo tiene como objetivo describir y relacionar los campos de tensión que existen en la gestión de la asesoría educativa. Los campos de tensión son aquellas situaciones de la asesoría educativa en las que se pueden encontrar dos extremos de interpretación. Para establecer los aspectos esenciales del estudio se realizó una investigación documental y una encuesta voluntaria a 30 asesores. Los campos de tensión son identificados con los siguientes criterios: requerimientos del sistema, intencionalidad, contexto, ámbito, enfoque, característica y permanencia. Se analiza antinómicamente: nivel central-nivel local, control-cambio educativo, escuelas iguales-escuelas diferentes, acompañamientoevaluación, paradigma cualitativo-paradigma cuantitativo, verticalidad-horizontalidad y desaparición-estabilidad. La conclusión principal es que el nivel central predomina sobre el nivel institucional educativo en los aspectos pedagógicos y administrativos. Se define que es posible lograr equilibrios entre los diferentes campos de tensión identificados. También que la asesoría educativa es el equipo humano que puede lograr este balance a través de su gestión técnica de alto nivel y experticia. La prospección es que los decisores políticos del sistema afiancen la asesoría junto con su función complementaria que es la auditoría educativa.

Palabras clave:

Asesoría, estándares, gestión, modelo, orientación, tensión 


\section{Abstract}

Educational counseling in Ecuador emerges in 2013 as a function that replaces school supervision. There are practically no studies or analyses of this important function within the organizational system of the Ministry of Education. This article aims to describe and relate the areas of tension that exist in the management of educational advising. Fields of tension are those situations in educational counseling in which two extremes of interpretation can be found. In order to establish the essential aspects of the study, a documentary investigation and a voluntary survey of 30 advisors were carried out. The fields of tension are identified with the following criteria: system requirements, intentionality, context, scope, focus, characteristic and permanence. It is analyzed antinomically: central-local level, control-educational change, equal schools-different schools, accompaniment-evaluation, qualitative paradigm-quantitative paradigm, verticality-horizontality and disappearance-stability. The main conclusion is that the central level predominates over the institutional educational level in the pedagogical and administrative aspects. It is defined that it is possible to achieve a balance between the different fields of tension identified. It is also defined that the educational consultancy is the human team that can achieve this balance through its highlevel technical management and expertise. The idea is that the political decision makers of the system will strengthen the consultancy together with its complementary function, which is the educational audit.

Key words

Advice, standards, management, model, orientation, tension

\section{Introducción}

La asesoría educativa es una función que, junto con la auditoría educativa, reemplazó a la supervisión escolar. Como parte de este proceso las autoridades ecuatorianas presentaron en 2009 un proyecto de Ley de Educación como lo relatan Aguerrondo y Xifra (2012a): “Una de las propuestas es la redefinición del marco para la tarea de los supervisores educativos surgida de las necesidades de modernizar el Estado y de un profundo cuestionamiento social de parte de estos agentes estatales." (p. 5). Las reformas estatales incluían una reorganización de la administración pública en un considerable esfuerzo de desconcentración, distribución territorial y gestión gubernamental.

La asesoría orienta, acompaña y realiza un seguimiento formativo a la gestión pedagógica de las instituciones educativas fiscales y fiscomisionales. Estas actividades centrales de la asesoría exponen la intención del Estado de transformar el sistema educativo en materia de acompañamiento y evaluación pedagógica. Así, "se exterioriza en la sustitución oficial del antiguo sistema de supervisión educativa, característico del modelo escolar centralizadoregulado, por un nuevo sistema, conocido como de 'apoyo y seguimiento a la gestión educativa', que es consistente con el modelo autonómico-regulado." (Cevallos, 2016, p. 69). Dentro del conjunto de reformas del sistema, las referentes a la asesoría suponen un cambio de rumbo substancial en el apoyo ministerial a las instituciones educativas.

En tanto función emergente dentro del sistema educativo, no existen análisis o investigaciones de este aparecimiento organizacional. Por ello la pregunta de partida es: ¿Qué aspectos caracterizan el surgimiento y desarrollo inicial de la asesoría educativa en el Ecuador? El nacer de la asesoría educativa está sujeto a los rigores propios de un elemento institucional del Estado. Han estado presentes una serie de eventos contrapuestos en el contexto político de su origen y progreso temprano. En este proceso de cambio se establece situaciones de contradicción que ubicados en dentro de dos polos de interpretación se los denomina campos de tensión. Gómez et al. (2017) señalan que el rasgo esencial de esta transformación se constituye en una especie de "oxímoron de la teoría política" (p. 11) en

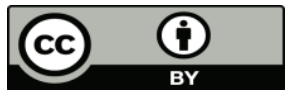

Licencia Creative Commons Atribución 4.0 Internacional (CC BY 4.0)

Revista Cátedra, 3(3), pp. 88-110, septiembre-diciembre 2020. e-ISSN: 2631-2875

https://doi.org/10.29166/catedra.v3i3.2406 
donde se entrecruzan una serie de aspectos confrontados e incluso paradójicos. La identificación de estos campos de tensión, establecer sus características y correlacionarlas, es la problemática que aborda el artículo.

Indispensable en una realidad de equipos directivos institucionales heterogéneo en lo profesional y académico, que requiere del apoyo constante por parte del Ministerio de Educación. Metodológicamente, el artículo es de carácter descriptivo y relacional.

El principal desafío de esta investigación es el establecimiento de los criterios para la caracterización de la asesoría educativa en su surgimiento. La teoría pedagógica y política generalmente exponen la confrontación entre el ámbito central y local como el principal punto de tensión de un sistema educativo. Identificar otros aspectos de oposición, colocarlos al mismo nivel del área de tensión del ámbito y desentrañar un área poco reflexionado es parte del reto. Indagar sobre procesos del sistema educativo que lo genera el ministerio del ramo cuenta con la dificultad del acceso a la información oficial. Ello se puede superar con la utilización de la documentación pública que emite el organismo estatal en especial su normativa adjetiva y sustantiva. La investigación de asuntos estatales siempre es sensible al decisor político que lidera los procesos como en el caso de la estructura educativa. Esta sensibilidad puede ser un óbice real, aunque innecesario, para la investigación académica.

El artículo contiene una visión retrospectiva de la asesoría educativa desde la función de inspectoría y supervisión escolar. Como función que emerge en el sistema sintetiza algunos aspectos legales y de gestión actual que le caracteriza. La sección principal aborda los siete campos de tensión identificados. Este abordaje es bibliográfico y con la presentación de los resultados de la encuesta que visualiza la realidad desde el propio equipo de asesoría educativa. Se presentan las respectivas conclusiones que se obtienen en relación a la investigación y de alguna manera evocan la prospectiva de la función.

\section{Consideraciones generales}

\subsection{Antecedente remoto: las inspectorías educativas}

El Ecuador adoptó su modelo educativo fundamentalmente desde la vertiente europea por su característica histórica de ser colonia española. Aquí se impusieron desde ese tiempo programas de carácter enciclopedista y libresco. Todos los atisbos de educación en la época colonial estuvieron bajo el signo de la religión cristiana. "Hubo una tendencia alienante y autoritaria dirigida a sostener a la corona y el medio que la iglesia tenía para imponer su credo." (OEI, s.f., p. 5). Esta disposición se mantuvo aun con la independencia como Estado en 1830. Sería con la revolución liberal a partir de 1895 y siguientes décadas que se lograron transformaciones importantes en el quehacer educativo.

Los cambios político-culturales de la Vieja Europa, inscritos en la "Edad de las Luces" variaron las ideas sociales en el Ecuador y, de hecho, produjeron variaciones en el pensamiento acerca de la educación y su práctica institucional. La posición radical del liberalismo, sus triunfos en la lucha por un nuevo poder político, determinaron que el hecho educativo sea responsabilidad del Estado y que tenga una concepción laica y democrática; sin embargo, no hubo cambios en la condición clasista de la enseñanza: el rasgo enciclopedista se reflejó en la instrucción europeizante que recibían las minorías detentadoras del poder político y de la economía (OEI, s.f., p. 6). 
Aguerrondo (2013) refiere que "los orígenes históricos de la función de inspección escolar se remontan al surgimiento de los sistemas educativos modernos y a la conformación de los estados nacionales, procesos ocurridos desde mediados del siglo XIX." (p. 4). Concretamente, la figura del «inspector general de estudios» aparece por primera vez en la legislación francesa del año 1802. Desde su inicio, esta función ha tenido tres tareas: control, apoyo y vinculación. El control en función de los diferentes aspectos de lo administrativo y organizacional de la institución educativa. El apoyo en términos de un asesoramiento dado desde una jerarquía superior y externa a cada centro escolar. La vinculación por constituirse en correa de transmisión del nivel central a los niveles intermedio y local de la gestión educativa. La perspectiva era que "a través del cumplimiento de estas tres tareas la supervisión llevaría al mejoramiento de la calidad de la educación" (Carron y De Grauwe, 2003 , p. 5). Este enfoque fue el principio fundamental que orientó la actividad de la inspección educativa en Europa y los países de su órbita pedagógica como el Ecuador.

\subsection{Referentes históricos en el Ecuador}

La asesoría educativa tiene sus raíces históricas en la inspección y supervisión educativa, como elemento de importancia dentro del sistema ecuatoriano. En los albores de la república, concretamente en el régimen de Vicente Rocafuerte (1934-1938) se expide el decreto del primer Orgánico de Enseñanza Pública. Esta norma establece la Dirección General de Estudios (antecedente del Ministerio de Educación) y las Subdirecciones e Inspectorías de Instrucción. El primero como organismo regulador y los segundos como instancias encargadas de cumplir y hacer cumplir las regulaciones (OEI, s.f., p. 1).

Posteriormente, la Ley Orgánica de Instrucción Pública de 1906, plena época de emergencia liberal, dispone dentro de los organismos a nivel provincial la "Visitaduría Escolar". La función del Visitador Escolar, "era coercitiva y tenía como objetivo vigilar, fiscalizar, comprobar y castigar las deficiencias de los maestros, sin ofrecerles ningún tipo de orientación" (Piruch, 2005, p. 30). Esto en una época con carencias de todo tipo. El Informe a la Nación del Ministro de Instrucción Pública del año 1912, describe esta realidad:

No tenemos locales adecuados, ni tenemos útiles de enseñanza, no tenemos personal idóneo, ni textos, no tenemos programas, no tenemos un sistema pedagógico racional y directo que haga de la escuela lo que debe ser: un estimulante y vigorizador del alma infantil, un centro atrayente donde el niño encuentre algo como una función de la labor de su desenvolvimiento físico, moral e intelectual. Cuartos obscuros, destartalados, antihigiénicos, en los que el niño se siente como deprimido, asfixiado y que no encuentra, por tanto, asiento sino un tosco banco, cuando no un adobe o el suelo de tierra; maestros gruñones, cuya ardua misión consiste en hacer repetir en coro el silabario y en caer a puñetazos sobre el muchacho que cometió el crimen de distraerse un momento; lecciones forzadas, monótonas, continuadas, abominables cuanto entorpecedoras. (OEI, s.f., p. 5)

Desde 1938 a 1966 se tiene la "Inspección Escolar" como organismo provincial dentro del Ministerio de Educación Pública (OEI, s.f., p. 3). Esta denominación da cuenta de un desarrollo dialéctico de la función. Piruch (2005), precisa que:

los Inspectores Escolares se esfuerzan por transformar su función, convirtiéndola en una tarea de orientación y ayuda. Desarrolla su labor

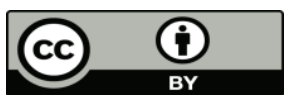

Licencia Creative Commons Atribución 4.0 Internacional (CC BY 4.0)

Revista Cátedra, 3(3), pp. 88-110, septiembre-diciembre 2020. e-ISSN: 2631-2875 https://doi.org/10.29166/catedra.v3i3.2406 
en forma más afable y cordial, menos imperativa, aunque persiste la tendencia a la autosuficiencia y la convicción de que todas las decisiones deben venir desde arriba. En definitiva, había cambiado la actitud, el modo de actuar, pero los procedimientos seguían siendo casi iguales a los de los visitadores (p. 24).

A partir de 1966 asume el nombre de "Supervisión Educativa" en la perspectiva de tener un rol distinto a los anteriores. Por supuesto, hay cambios importantes. Sin embargo, la función mantiene el modelo jerárquico-piramidal del Estado que impone una organización vertical y burocrática del sistema educativo. Esta estructura "no ha permitido la adecuación de la enseñanza a las necesidades singulares de los beneficiarios y diferentes sectores" (Aguerrondo y Xifra, 2012a, p. 5). El Modelo Nacional de Supervisión Educativa preveía las funciones de quien supervisaba como contralor, mediador y coordinador de los aspectos técnico, pedagógico y administrativo. Estas atribuciones se ejecutaban mediante el monitoreo, evaluación, acompañamiento y asistencia a las instituciones. Es decir, de modo puntual un supervisor o supervisora tenía tres funciones: 1) Auditoría, 2) Asesoría, y, 3) Mediación.

El modelo y la función de supervisión se agotó en el sistema educativo ecuatoriano. Los cambios requeridos tanto desde la estructura como de las políticas nacionales definidas en el Plan Decenal de Educación 2006-2015, impusieron una transformación de esta instancia. Aguerrondo y Xifra (2011) señalan que:

El Ministerio de Educación trabajó en la propuesta de una nueva ley de educación que envió a la Asamblea Legislativa en septiembre de 2009 y fue aprobada en enero de 2011. Una de las propuestas más importantes en esta nueva ley tiene que ver con un cambio sustantivo en el modelo de la supervisión educativa ya que propone la supresión de la figura de los supervisores y su reemplazo por dos roles diferenciados: los asesores por un lado y los auditores por el otro (p. 4).

Ante su desgaste en el conjunto del sistema, la transformación de la supervisión en asesoría y auditoría educativa fue inevitable. El proceso involucró a la función ejecutiva y legislativa del Estado ecuatoriano por la complejidad del tema y los actores involucrados. La transición incluyó que varios supervisores derivaran hacia la nueva función previa evaluación acordes al perfil requerido.

Otro referente histórico importante de la asesoría en el Ecuador es el de la supervisión educativa del Modelo de Educación Intercultural Bilingüe instituida a partir de 1988. Como define Piruch (2005): "la Supervisión Educativa Intercultural Bilingüe, es un organismo técnico asesor, que define las políticas socio-organizativas, técnicas pedagógicas y administrativas, encaminadas al cumplimiento de los principios, fines y objetivos del Sistema de Educación Intercultural bilingüe" (p. 46). Si bien mantiene las tres funciones que tiene la supervisión educativa general del país, en este modelo se enfatizó en la función de asesoría y apoyo.

EL apoyo técnico pedagógico que se realizaba en educación inicial también se constituye en el otro referente de la asesoría educativa. El servicio para atender a la población estudiantil menor a los seis años de edad nació a la luz de la revolución alfarista a inicios del siglo XX. Pautasso (2009), precisa que, "la incorporación del liberalismo como sistema nacional, la 
adopción del laicismo como eje fundamental de la educación, la emancipación e ingreso de la mujer en el mercado laboral e intelectual, la mayor concentración de niños, el desarrollo de cierta conciencia en cuanto a aprovechar el potencial del infante" (p. 5), contribuyó al origen de la educación parvularia. A finales del siglo XX, las modalidades escolarizadas denominadas jardines de infantes o incorporadas a otras instituciones educativas, recibían el apoyo pedagógico de supervisoras especializadas en su mayoría. En las modalidades no escolarizadas se tenía una asistencia técnica y pedagógica de parte de docentes con especialización y experiencia en el trabajo con infantes. Estas modalidades no escolarizadas incluían el Programa Nacional de Educación Preescolar - PRONEPE, Programa Operación Rescate Infantil (ORI) y el Programa de Desarrollo Infantil (INNFA).

\section{La asesoría educativa: función emergente}

\subsection{Descentralización y calidad educativa}

Las referencias más generales de la asesoría educativa están en la Ley Orgánica de Educación Intercultural - LOEI (Ministerio de Educación, 2016). Dentro de las competencias del Consejo Académico del Circuito Educativo Intercultural y o Bilingüe: "Art. 31.- Impulsar la calidad educativa en establecimientos del circuito conjuntamente con asesores y auditores educativos" (p. 35). Como una de las funciones de la carrera docente pública: "Art. 114.- Los profesionales de la educación podrán ejercer la titularidad de las siguientes funciones: f) Asesores educativos" (p. 64).

En la legislación sustantiva, se tienen varias disposiciones en el Reglamento General a la Ley Orgánica de Educación Intercultural (2015): en el conjunto de atribuciones del Subdirector o Vicerrector de una institución educativa: “Art. 45.- (numeral) 5. Asesorar y supervisar el trabajo docente" (p. 16). Determina la función principal de la asesoría: "Art. 309.- orientar la gestión institucional hacia el cumplimiento de los estándares de calidad educativa definidos por el Nivel Central de la Autoridad Educativa Nacional" (p. 89). El Modelo Nacional de Apoyo y Seguimiento a la Gestión Educativa (MNASGE, 2013), especifica las funciones en correlación a la normativa señalada:

1. Asesoramiento y orientación para la implementación, desarrollo y ejecución curricular.

2. Orientación de actividades de innovación y cambio educativo.

3. Comunicación y coordinación pedagógicas.

4. Acompañamiento y seguimiento a los procesos pedagógicos y de gestión (p. 9).

Todas estas determinaciones legales y técnicas se deben entender como las definiciones de una función emergente en el Ecuador. En los demás países de América Latina, la asesoría educativa sigue como parte de las funciones de la supervisión educativa. En el caso de México, por ejemplo, la asesoría educativa existe también separada como un servicio estatal, pero bajo el nivel de la supervisión (Antúnez, 2012 y López, 2010). Así, en el continente, el modelo ecuatoriano se constituye en pionero en separar las funciones de control y apoyo.

El nuevo modelo de gestión educativa incluye cinco niveles como muestra el cuadro 1 . La actividad esencial de los actores en cada nivel lo define el estatuto orgánico de gestión organizacional por procesos (Ministerio de Educación, 2017a). Estas actividades muestran el principio de un Estado rector y de instancias menores de coordinación y ejecución que cumplan un papel específico cercano a la autonomía. Expresan en la práctica la posibilidad de transformar la estructura del sistema educativo en su arquitectura. Cevallos (2016) señala:

Licencia Creative Commons Atribución 4.0 Internacional (CC BY 4.0)

Revista Cátedra, 3(3), pp. 88-110, septiembre-diciembre 2020. e-ISSN: 2631-2875

https://doi.org/10.29166/catedra.v3i3.2406 
5. la arquitectura institucional de un sistema escolar se refiere a la manera como están organizadas estructuralmente las escuelas de ese sistema, lo que incluye las relaciones de poder y de otro tipo que se establecen entre los actores de las escuelas (principalmente docentes y directivos), los representantes del Estado -nacional, regional o local- y otros actores del sistema, tales como los estudiantes y sus familias, las asociaciones gremiales y patronales relacionadas con la educación y otros miembros y sectores de la sociedad (p. 57).

Los sistemas educativos exitosos, entre otros factores de su mejora, han desarrollado un nivel de mediación entre las instituciones educativas y el nivel central. Por supuesto, en algunos países esta intermediación viene acompañada de control y evaluación como la hacen las inspectorías o supervisión. En el Ecuador, la función muy bien definida para esta función es la asesoría educativa. Mourshed, Chijioke y Barber (2012) precisan:

los sistemas educativos que analizamos progresan en su trayecto de mejora, parecen depender cada vez más de un "nivel intermediario" que actúa entre el centro y las escuelas. Este nivel intermediario sostiene la mejora otorgando tres aspectos importantes al sistema: respaldo práctico y específico a las escuelas, un amortiguador entre la escuela y el centro, y un canal para compartir e integrar las mejoras entre las escuelas (p.18).

\begin{tabular}{ll}
\hline \multicolumn{1}{c}{ NIVEL DEL SISTEMA } & \multicolumn{1}{c}{ ACTIVIDAD ESENCIAL } \\
\hline Autoridad Educativa & $\begin{array}{l}\text { Garantiza y asegura el cumplimiento del derecho a la } \\
\text { educación en los cuatro niveles de gestión. } \\
\text { Diseñar y actualizar sistemas para la asesoría educativa en } \\
\text { los ámbitos administrativo y pedagógico, en los niveles } \\
\text { desconcentrados } \\
\text { Coordinar y aplicar las políticas, normativas, estrategias, } \\
\text { planes y programas para la asesoría educativa. }\end{array}$ \\
Nivel Distrital (147 & $\begin{array}{l}\text { Ejecutar las políticas, normativas, estrategias, planes y } \\
\text { programas para la asesoría de la gestión educativa. }\end{array}$ \\
Distritos) & $\begin{array}{l}\text { Ejecutar las políticas, normativas, estrategias, planes y } \\
\text { programas para la asesoría de la gestión educativa. }\end{array}$ \\
Circuitos) & Asesorar y supervisar la labor docente. \\
Nivel institucional &
\end{tabular}

Cuadro 1: Acciones esenciales de Asesoría Educativa

La constatación empírica constata la necesidad de que exista un mediador entre el ministerio y los centros escolares para lograr la mejorar la gestión en el aula. La literatura pedagógica ratifica este aserto bajo la premisa de que si bien las instituciones educativas son el factor primordial para mejorar la calidad de los aprendizajes "por sí solas no son suficientes para lograr este objetivo de manera sostenible." (Cevallos, 2016, p. 57). Es indispensable "ayudar a las escuelas con conocimientos obtenidos a partir de estudios científicamente validados y de experiencias llevadas a cabo por otros centros para poner en marcha y desarrollar con éxito procesos de cambio que les permitan conseguir mejor sus objetivos." (Muñoz y Murillo, 2010, p. 8). La gestión pedagógica mejorada en las instituciones educativas depende de sus propias fortalezas internas y del apoyo externo que le puede brindar la asesoría. Siempre hay que considerar que este respaldo externo incluye también a la función de auditoría educativa que realiza la evaluación institucional. Las dos 
funciones son las previstas en el Nuevo Modelo Nacional de Apoyo y Seguimiento que sustituyó a la supervisión educativa.

\subsection{Situación nacional}

El proceso de transición de la supervisión a la asesoría educativa se ratifica el 2011 con la aprobación de la LOEI (Ministerio de Educación, 2016). En la práctica se inicia con la incorporación de equipos de asesoría educativa en las zonas 6, 7, 8 y 9 del Ecuador. Para el 2018 ya se habían ubicado equipos en las demás zonas del país. Sin embargo, hay significativa diferencia entre lo proyectado y lo ejecutado en relación a la dotación del talento humano requerido en dichos equipos. En el cuadro 2 se puede constatar estadísticamente estas diferencias y la situación numérica de asesoría educativa.

Conforme al Acuerdo 557-2012 de la Secretaría Nacional de Planificación y Desarrollo (SENPLADES, 2012) se conforman "140 distritos administrativos de planificación, así como 1134 circuitos administrativos de planificación, a nivel nacional, para la gestión de las entidades y organismos que conforman la Función Ejecutiva" (p. 2). El diseño inicial de conformación del equipo nacional de asesoría educativa preveía dos asesores por cada circuito, que da un total de 2260. Hasta fines de 2019 se tenían 146 funcionarios que equivale al 6,5\% de cumplimiento. Las zonas administrativas 8 y 9, Guayaquil y Quito, eran las más dotadas con el 21,6 \% y 14,7 \%. Las menos atendidas, con el 1,9 \% y 2,9\%, respectivamente, eran la Zona 4 (Manabí y Santo Domingo de Los Tsáchilas) y Zona 5 (Bolívar, Guayas, Los Ríos, Santa Elena y Galápagos).

\begin{tabular}{cccccc}
\hline Zona & $\begin{array}{c}\text { Nro. de } \\
\text { distritos }\end{array}$ & $\begin{array}{c}\text { Nro. de } \\
\text { circuitos }\end{array}$ & $\begin{array}{c}\text { Número proyectado } \\
\text { (Número de circuitos x 2) }\end{array}$ & $\begin{array}{c}\text { Número } \\
\text { ejecutado }\end{array}$ & $\begin{array}{c}\text { Porcentaje } \\
\text { ejecutado }\end{array}$ \\
\hline 1 & 16 & 139 & 278 & 9 & $3,2 \%$ \\
2 & 8 & 58 & 116 & 9 & $7,8 \%$ \\
3 & 19 & 142 & 284 & 11 & $3,9 \%$ \\
4 & 15 & 155 & 310 & 6 & $1,9 \%$ \\
5 & 25 & 192 & 384 & 11 & $2,9 \%$ \\
6 & 17 & 120 & 240 & 25 & $10,4 \%$ \\
7 & 19 & 165 & 330 & 19 & $5,8 \%$ \\
8 & 12 & 67 & 134 & 29 & $21,6 \%$ \\
9 & 9 & 92 & 184 & 27 & $14,7 \%$ \\
No delimitado & 4 & & & \\
\multicolumn{7}{l}{ Total } & 140 & 1134 & 2260 & 146 & $6,5 \%$ \\
\hline
\end{tabular}

Cuadro 2: Número y porcentaje de asesores educativos proyectado y ejecutado

Al escaso número de asesores educativos, se debe añadir su derivación hacia diversas actividades determinadas por el Nivel Central de la Autoridad Educativa Nacional. Desde su origen, dentro del sistema, cumplieron actividades distintas a las de asesoría. Fueron apoyo en los procesos de inscripciones que desde el 2014 se realizan mediante plataformas tecnológicas por el Ministerio de Educación. Actuaron como soporte en la titulación de bachilleres. Orientaron a coordinadores y operadores en sedes para traslados de estudiantes que se derivan al sistema fiscal. Cumplieron funciones de interventores educativos en aquellas instituciones que entraron en situaciones de complejidad 
organizacional y de gestión. Han cumplido y cumplen funciones administrativas a nivel de coordinación zonal, unidades departamentales del ministerio, direcciones distritales. Fueron y son contraparte del Ministerio de Educación ante diversos organismos gubernamentales y no gubernamentales. La mayoría de estas actividades y otras que se ejecutaron, están relacionadas a la regulación educativa. A partir del 2018, la derivación fue menos frecuente y los equipos pudieron enfocar su gestión dentro de las funciones específicas previstas para la asesoría educativa.

\subsection{Acción de la asesoría educativa}

La asesoría es un proceso de orientación y acompañamiento a la gestión educativa en la perspectiva de que las instituciones alcancen los estándares de calidad. El MNASGE (2013) diseñado por el Ministerio de Educación la considera como "una acción técnica de carácter profesional cuya función principal es orientar la gestión institucional" (p. 9). Este proceso general de orientación se subdivide en procesos específicos previstos en el Manual de Asesoría Educativa (Ministerio de Educación, 2014). El primero corresponde a la planificación que contiene como actividades la "preparación, identificación, elaboración y aceptación de solicitud, organización de las actividades de asesoría y planificación de la agenda" (p. 10). El segundo subproceso es el asesoramiento en sí y que implica entre sus acciones al "diagnóstico situacional, planificación de la asesoría, acompañamiento y monitoreo a la implementación y evaluación de resultados" (p. 10). Finalmente, el ciclo se cierra con la evaluación que incluye "valoración, análisis de resultados y actividades de desarrollo profesional" (p. 10). Este manual se constituye en uno de los principales referentes que tienen los equipos de asesoría para el desarrollo de su función.

El proceso general descrito se refiere a la asesoría educativa externa al centro escolar. Por razones de la emergencia de la función, la figura simbólica de la asesoría está percibida en el equipo de profesionales que envía el ministerio a la institución. Sin embargo, como ya se estableció en la base legal, la asesoría educativa también es atribución de las principales autoridades de una institución. Estos elementos contribuyen a una tipificación de la asesoría educativa en externa e interna. La externa que corresponde a la labor que diseña, ejecuta y evalúa el equipo de profesionales designados para el efecto por la autoridad educativa. La interna que referencia a la atribución del rectorado o dirección escolar en el ámbito de su apoyo al personal docente. En cualquiera de los casos, el principio es que se entiende a la institución educativa como una unidad organizacional, administrativa y pedagógica. Esta noción de totalidad conlleva a que la asesoría educativa externa o interna dirija su esfuerzo al cumplimiento de los estándares de gestión escolar. Por ello, para el Ministerio de Educación (2017b), estos estándares:

se aplican a los establecimientos educativos. Se refieren a los procesos de gestión y prácticas institucionales que contribuyen al buen funcionamiento de la institución. Además, favorecen el desarrollo profesional de las personas que conforman la institución educativa permitiendo que esta se aproxime a su funcionamiento ideal (p. 14).

Los estándares de gestión escolar determinan el accionar de los equipos de asesoría. En la dimensión de gestión administrativa tiene que ver con la organización institucional, el desarrollo profesional de autoridades y docentes, la información y comunicación institucional y la infraestructura, equipamiento y servicios complementarios. En la segunda dimensión, la pedagógica, se tiene el componente enseñanza y aprendizaje y el de consejería 
estudiantil y refuerzo pedagógico. La tercera dimensión se refiere a la convivencia y participación escolar y a las alianzas estratégicas de cooperación para el desarrollo. La cuarta dimensión corresponde a la gestión de riesgos y protección.

En lo práctico, la asesoría educativa externa realiza el acompañamiento a los cuerpos directivos en la construcción de diversas herramientas de la planificación estratégica. Estas herramientas se elaboran, en lo ideal, con la participación de toda la comunidad educativa bajo el liderazgo de las autoridades. El equipo asesor debe estar implicado en los dos aspectos que implica la construcción de los elementos de planificación estratégica: los procesos y los productos. Involucra el acompañamiento a las autoridades del centro educativo en el diseño, elaboración, aplicación y evaluación de tres instrumentos esenciales: Proyecto Educativo Institucional, Planificación Curricular Institucional y Código de Convivencia. La orientación del equipo asesor incluye elementos como la atención a estudiantes con Necesidades Educativas Especiales con adaptaciones curriculares. También el apoyo a la capacitación interna en algunas instituciones y la construcción de documentos de procedimientos administrativos y pedagógicos. Un aspecto importante que ayuda al trabajo del equipo asesor externo es la implementación del trabajo cooperativo en redes institucionales.

La acción del equipo de asesoría externo en las instituciones educativas tiene como punto de partida tres motivaciones establecidas. La primera es la atención a aquellas instituciones que tienen informe de auditoría educativa. En este caso se analizan los puntos críticos encontrados y se elabora un plan de mejora con los procesos y productos a cumplirse. La segunda es la solicitud institucional en la que orienta en los puntos específicos señalados en la petición. La tercera son aquellos procesos que genera la coordinación zonal o subsecretarías y la Dirección Nacional de Asesoría a la Gestión Educativa - DNAGE.

La visita de acompañamiento implica un protocolo de actuación que se halla establecido en el Código de Ética definido por la DNAGE (Ministerio de Educación, 2014). Los principios éticos establecidos son: "comportamiento profesional, objetividad, confidencialidad, cooperación y lealtad, criticidad, iniciativa” (p. 8). El comportamiento profesional implica la expresión de los siguientes valores éticos: "libertad y responsabilidad, honestidad, puntualidad, tolerancia, justicia, servicio, observancia y respeto a la norma, y compromiso" (p. 7). La aplicación concreta de estos principios y valores éticos brinda confianza en los actores y sujetos de la asesoría en los distintos niveles del sistema.

\section{Metodología}

El artículo, en lo metodológico, es de tipo descriptivo pues "busca especificar las propiedades, las características y los perfiles importantes de personas, grupos, comunidades o cualquier otro fenómeno que se someta a un análisis" (Cortés y León, 2004, p. 20). Implica una investigación documental de aspectos históricos y actuales sobre la asesoría conocida en otros países como parte de la inspectoría o supervisión escolar. En lo histórico, se rastrea sintéticamente la trayectoria de esta función a lo largo de su presencia en las estructuras educativas a nivel nacional. Involucra un análisis de la legislación adjetiva y sustantiva alrededor de la función asesoría educativa dentro del sistema ecuatoriano. El aspecto actual determina la configuración del sistema educativo en diferentes niveles de autogestión en lo correspondiente al tema. Se realizó una encuesta anónima y voluntaria a treinta asesores en servicio activo y desvinculados por jubilación a escala nacional. De este modo se obtuvo la percepción de este grupo profesional sobre los campos de tensión identificados y anclar la reflexión en su realidad.

Licencia Creative Commons Atribución 4.0 Internacional (CC BY 4.0)

Revista Cátedra, 3(3), pp. 88-110, septiembre-diciembre 2020. e-ISSN: 2631-2875

https://doi.org/10.29166/catedra.v3i3.2406 


\section{Resultados y discusión}

\subsection{Los campos de tensión de la asesoría educativa}

Los campos de tensión son aquellas situaciones de la asesoría educativa en las que se pueden encontrar dos extremos de interpretación. Si bien se presentan como antípodas en términos que se contraponen claramente, sus diferencias no deben ser entendidas como insalvables. Más bien, estas situaciones son antinomias: "contradicción entre dos preceptos legales o entre dos principios racionales" (RAE, 2019). Estas contraposiciones son dinámicas y van a depender del tiempo y espacio de su aplicación para que se expresen hacia uno de los polos de interpretación. Esta dinamia a la vez permitiría, en términos generales, equilibrios en la perspectiva de lograr un balance en la gestión de la asesoría educativa. Los campos de tensión con los criterios para su definición se encuentran en el Cuadro 3.

\begin{tabular}{|c|c|c|}
\hline CAMPO & CRITERIO & OPOSICIÓN \\
\hline Campo de tensión 1 & $\begin{array}{l}\text { Requerimientos } \\
\text { del sistema }\end{array}$ & Nivel central versus Nivel local \\
\hline Campo de tensión 2 & Intencionalidad & Control versus Cambio educativo \\
\hline Campo de tensión 3 & Contexto & Escuelas iguales versus Escuelas diferentes \\
\hline Campo de tensión 4 & Ámbito & Acompañamiento versus Evaluación \\
\hline Campo de tensión 5 & Enfoque & $\begin{array}{l}\text { Paradigma cualitativo versus Paradigma } \\
\text { cuantitativo }\end{array}$ \\
\hline Campo de tensión 6 & $\begin{array}{l}\text { Relación con } \\
\text { directivos }\end{array}$ & Verticalidad versus Horizontalidad \\
\hline Campo de tensión 7 & Permanencia & Desaparición versus Estabilidad \\
\hline
\end{tabular}

Standaert (2012) construye el marco de interpretación de la calidad de la educación en términos de la influencia de la política del nivel central con el del nivel local. "Los sistemas de inspección se organizan a nivel central, desde donde se monitorea y garantiza la calidad de las escuelas a través de dicha función" (p. 31). La estructura educativa está diseñada en su conjunto nacional desde el nivel central a través de la legislación y de las políticas públicas que determina un gobierno. Esta estructura tiene un enorme peso e imposición en las definiciones que se hagan en el nivel educativo local. El currículo obligatorio (organización por áreas, objetivos, contenidos, etc.), subniveles educativos, estructura de organismos internos institucionales y muchos aspectos son determinados "desde arriba". Obviamente estas determinaciones establecen dinámicas administrativas y pedagógicas específicas en todos los demás niveles del sistema educativo.

Por el otro lado, hay que considerar la diversidad cultural y elementos del entorno económico y sociocomunitario a la institución educativa. Hacia lo interno, la cultura institucional está constituida por una heterogénea formación académica docente, el clima de convivencia, la capacidad de liderazgo y muchos otros factores. Desde la perspectiva del cambio y la profesión docente "las escuelas no son organizaciones donde se aprenda. En general no son lugares interesantes ni gratificantes para los docentes ni para los alumnos" (Fullan y Hargreaves, 1999, p. 12) Estos aspectos de la realidad institucional igual tienen su peso específico al momento de ejecutar las políticas y decisiones nacionales. La implementación a nivel local, para el Ecuador los niveles distritales, circuitales e institucionales, está sujeta a contingencias propias del contexto en la que se desenvuelve. 
Como se puede ver en el cuadro 4, los equipos de asesoría educativa externa reflexionan que mayoritariamente prevalecen las determinaciones del nivel central por sobre las del nivel institucional. Se debe considerar que en la regulación pública de los sistemas educativos se presentan tres efectos emergentes: "el 'efecto de contaminación' o diseminación de discursos, textos e ideologías; el 'efecto de hibridación' entendido como superposición y mestizaje de diferentes lógicas, discursos y prácticas en la definición y en la acción política; y el 'efecto mosaico' que permite identificar el cambio progresivo de la idea tradicional de sistema educativo" (Miranda et al, s.f., p. 1). Una explicación plausible es que se tiene una coyuntura, algo extendida en el tiempo, del surgimiento de la función asesora. Su posicionamiento en el sistema, que se debe considerar como innovación global, requiere del liderazgo estatal que solamente lo puede realizar el nivel ministerial. No hacerlo así implicaría la configuración de una función dispersa y sin identidad. Sin embargo, parte de dicha identidad, debería aceptar que la implementación curricular es de incumbencia institucional y requiere el apoyo en esa dirección.

\begin{tabular}{lc} 
En la función de asesoría educativa prevalece las determinaciones del nivel \\
central del Ministerio de Educación por sobre las necesidades del nivel \\
institucional (escuelas, colegios). \\
\hline Totalmente de acuerdo & $40 \%$ \\
De acuerdo & $43 \%$ \\
Ni de acuerdo ni en desacuerdo & $7 \%$ \\
En desacuerdo & $7 \%$ \\
Totalmente en desacuerdo & $3 \%$ \\
\hline
\end{tabular}

Cuadro 4: Campo de tensión 1: Nivel central versus Nivel local

El segundo campo es que la acción de la asesoría educativa se convierta en "agencia de control o en factor de cambio educativo" (Velásquez, 2011, p.1). En el extremo de constituirse en elemento de control se tendría en una función que enfatice lo administrativo por sobre lo pedagógico. Se caracterizaría por una acción fiscalizadora y punitiva que enfatice en la comprobación de la manera cómo se aplica las normativas legales vigentes. Esta verificación, por delegación expresa de la ley, supone la confirmación del funcionamiento escolar, especialmente sobre los directivos y docentes. La consecuencia de este control conduce al ejercicio de otra función más amplia que es el de la evaluación institucional" (Casanova, 2005, p.3). El exceso de control heteronomiza la gestión institucional, crea dependencia y prácticamente disminuye las iniciativas y desarrollo administrativo y pedagógico propias de una institución. Como agente de cambio, la asesoría es el servicio que está en mejores condiciones para garantizar un apropiado desarrollo de calidad de las instituciones. Ello a través del conocimiento de la normativa legal vigente y su cumplimiento contextualizado. Los equipos de asesoría tienen dominio conceptual y empírico en los diferentes ámbitos de la gestión administrativa y pedagógica. Dichas experticias redundan en ser orientadores hacia el cambio de una dinámica institucional que requiere renovarse para su desarrollo. Incluye una influencia que puede lograr renovadas capacidades en los actores institucionales. Este extremo también aplica a la capacidad de mediación entre los diferentes niveles de gestión del sistema que tiene la asesoría educativa. En el Campo de Tensión 2, expresado cuantitativamente en el cuadro 5, se establece que los equipos de asesoría educativa externa piensan en su gran mayoría que son los aspectos de control indagados los que predominan. Tiene concordancia con el Campo de Tensión 1, pues al prevalecer el nivel central se entiende que haya el énfasis en comprobar la implementación de la normativa. Muñoz (2017) precisa: 
En las sociedades de control aparece un nuevo orden cuyo régimen de dominación tiende a optimizar y aumentar en sutilidad aquellas formas de poder del régimen disciplinario. En ellas, el individuo ya no necesita encontrarse en una institución cerrada para verse sometido a determinadas tecnologías de poder: Las paredes de las instituciones se desploman; de modo que resulta imposible distinguir entre el interior y el exterior (p. 325).

Por ello, un elemento que pesa es que las autoridades y docentes de las instituciones educativas miran a los equipos de asesoría educativa como la anterior supervisión. Hay que considerar que por la estructura de la encuesta se preguntó sobre un polo del campo de tensión y no del otro.

En la función de asesoría educativa predomina la comprobación de la manera cómo se aplica la normativa vigente y la confirmación del funcionamiento organizacional de la institución educativa.

\begin{tabular}{lc}
\hline Totalmente de acuerdo & $37 \%$ \\
De acuerdo & $47 \%$ \\
Ni de acuerdo ni en desacuerdo & $3 \%$ \\
En desacuerdo & $7 \%$ \\
Totalmente en desacuerdo & $7 \%$ \\
\hline
\end{tabular}

Cuadro 5: Campo de tensión 2: Control versus Cambio educativo

El tercer campo de tensión de la asesoría se produce por el contexto político-educativo que genera el binomio homogeneidad/diversidad institucional. Por un lado, el apoyo de un proceso o producto institucional busca garantizar que todas las escuelas y colegios alcancen los estándares de calidad educativa de un modo más o menos similar. Esto en cuanto al cumplimiento del currículo obligatorio, la organización mínima requerida en lo administrativo y pedagógico, definición de las mallas curriculares. Se propende que haya homogeneidad en la existencia de documentos y procesos estratégicos como el Proyecto Educativo Institucional, Plan Curricular Institucional y Código de Convivencia Armónica. Por el otro polo, el enfoque es contribuir que las instituciones educativas sean distintas. Esta distinción tiene su fuente en las diferencias de su población estudiantil o nivel educativo que tiene en su oferta. Toma en cuenta a los contextos comunitarios que rodean a la escuela/colegio, a los grupos socioculturales que atiende. Esta perspectiva atiende y fomenta las potencialidades institucionales hacia la relativa autonomía respecto de los niveles desconcentrados de los que forma parte. En este polo, los equipos de asesoría no cambiarán la función ni los estándares sino el tipo de proceso y contenido del acompañamiento.

Las respuestas de los asesores se dispersan en esta pregunta sobre la similitud/diferencia de las instituciones a las que apoyan como se puede ver en el cuadro 6. Si bien el $33 \%$ está "Totalmente de Acuerdo" y el 30 \% "De Acuerdo", hay otro 30 \% que está en "En desacuerdo" y "Totalmente en Desacuerdo". En conjunto se considera que lo esencial es que las instituciones alcancen los estándares por igual y simultáneamente sus productos y procesos sean adecuados a su contexto escolar. Es posible que en la interrogante haya sido entendida lo "similar" como un requerimiento general del sistema sin que necesariamente se tenga que desatender lo específico de la realidad institucional.

Para los equipos de asesoría la perspectiva es que las escuelas/colegios alcancen de la manera más similar posible los estándares de calidad educativa.

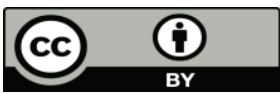

Licencia Creative Commons Atribución 4.0 Internacional (CC BY 4.0)

Revista Cátedra, 3(3), pp. 88-110, septiembre-diciembre 2020. e-ISSN: 2631-2875 https://doi.org/10.29166/catedra.v3i3.2406 
Totalmente de acuerdo

De acuerdo

$\mathrm{Ni}$ de acuerdo ni en desacuerdo

En desacuerdo

Totalmente en desacuerdo

Cuadro 6: Campo de tensión 3: Escuelas iguales versus Escuelas diferentes

El cuarto campo de tensión se origina cuando las funciones de evaluación (control) y asesoramiento de la supervisión/inspectoría educativa entran en contradicción. En el caso ecuatoriano, al tener separadas estas responsabilidades en equipos de funcionarios distintos, la situación incluso se presenta con mucha frecuencia. La asesoría implica un acompañamiento a los directivos institucionales en los diversos procesos dirigidos a la mejora en función de los estándares de calidad educativa. El inicio de este acompañamiento requiere de una evaluación institucional que no siempre es posible sea cumplido por el equipo de auditoría educativa. De modo general el equipo de asesoría también aplica un diagnóstico situacional previsto en su manual de procedimientos. El informe de evaluación institucional que emite el equipo de auditoría educativa, contiene una serie de recomendaciones. En estricto, esto corresponde a las funciones de la asesoría pero que el auditor no puede dejar de establecer con el carácter de sugerencias.

\begin{tabular}{lc}
\hline $\begin{array}{l}\text { El acompañamiento que realiza la asesoría educativa implica tener una evaluación } \\
\text { institucional realizada por el mismo equipo de asesores. }\end{array}$ \\
\hline Totalmente de acuerdo & $27 \%$ \\
De acuerdo & $30 \%$ \\
Ni de acuerdo ni en desacuerdo & $6 \%$ \\
En desacuerdo & $17 \%$ \\
Totalmente en desacuerdo & $20 \%$ \\
\hline
\end{tabular}

Cuadro 7: Campo de tensión 4: Acompañamiento versus Evaluación

La relación acompañamiento/evaluación se dispersa notoriamente como se puede ver en el Cuadro 7. El $57 \%$ acumulado está de acuerdo y el $37 \%$ en desacuerdo. La mayoría de escuelas/colegios fiscales no han sido auditadas y por ende no cuentan con su evaluación institucional. Esto se explica por el número de auditores y auditoras educativas que es significativamente menor que el de asesoría. Ello conlleva a que equipos de asesoría educativa desarrollen un diagnóstico situacional como lo prevé su manual y que eventualmente sea considerado como evaluación institucional. Por lo demás, la determinación de las funciones de asesoría y auditoría educativas están muy claramente especificadas en la normativa y en el diseño del MNASGE.

El quinto campo de tensión se presenta en la perspectiva de que la asesoría es esencialmente un proceso socioeducativo que requiere de investigación para contextualizar su desarrollo. En la academia se tiene amplia literatura sobre los paradigmas cualitativo y cuantitativo tanto en su fundamentación epistemológica como de los elementos metodológicos que los concretizan. Aquí se trata de realizar una aplicación específica en el contexto de la asesoría educativa ecuatoriana. En el accionar de asesoría enfatizar lo cuantitativo implica priorizar los parámetros de apoyo y acompañamiento en la planificación estratégica. Por ello, es de carácter deductivo. Enfatiza el número de visitas, la frecuencia de reuniones con autoridades institucionales, el número de procesos 
acompañamientos, entre otros aspectos de cantidad. Todo se transforma en datos numéricos: situaciones observadas, registros de atención, medidas de aplicación, logros y dificultades. El método cuantitativo da importancia a lo objetivo, la precisión de los procedimientos y la cuantificación en base a indicadores establecidos a través de variables. En lo cualitativo, la asesoría educativa basa su accionar en estricta relación a las características del grupo de autoridades, docentes y el entorno institucional. Por ello, la perspectiva cualitativa es de carácter inductivo. Trata de comprender al directivo escolar dentro de su propio escenario que se constituye en el marco de referencia para el desarrollo de los procesos de acompañamiento. La información se entrega como textos descriptivos, resultados narrativos de sus observaciones, explicaciones de entrevistas realizadas, entre otras formas.

En el Cuadro 8 se determina que el $57 \%$ de personas encuestadas manifiesta su acuerdo en dato acumulado. El 37 \% su desacuerdo a que prevalece lo objetivo por sobre lo subjetivo y lo general por encima de las especificidades. La presión de entregar información hacia los niveles superiores de la autoridad educativa nacional y otros estamentos estatales, es la motivación principal de este énfasis. Para la toma de decisiones en el nivel central y zonal se torna en insumo indispensable esta información de campo que la producen los equipos de asesoría. Esta demanda dentro del sistema impone que haya la percepción de prevalencia del paradigma cuantitativo por sobre el cualitativo. Sin embargo, un tercio de personas encuestadas, consideran que en su labor asesora es lo particular y las realidades institucionales las importantes como parte de su función.

\begin{tabular}{lc}
\hline $\begin{array}{l}\text { En la función de asesoría educativa prevalece lo objetivo por sobre lo subjetivo, las } \\
\text { de generalizaciones por sobre las especificidades. }\end{array}$ \\
\hline Totalmente de acuerdo & $27 \%$ \\
De acuerdo & $30 \%$ \\
Ni de acuerdo ni en desacuerdo & $6 \%$ \\
En desacuerdo & $17 \%$ \\
Totalmente en desacuerdo & $20 \%$ \\
\hline
\end{tabular}

Cuadro 8: Campo de tensión 5: Paradigma cualitativo versus Paradigma cuantitativo

El sexto campo de tensión tiene que ver con el cómo se percibe a la asesoría en su relación con los equipos directivos institucionales y docentes. En un extremo está la percepción de verticalidad que caracterizaría a la anterior supervisión educativa y cuyo remanente se mantiene en los actores institucionales. Al respecto, Marcos Santos (2005) precisa que:

La verticalidad como manera de pensar y percibir la realidad es, desde luego una ideología, en tanto que pensamiento legitimador de una sociedad caracterizada por la escisión y separación entre sus miembros. Implica la ordenación, a menudo inconsciente, que el sujeto hace de las demás personas, una ordenación vertical en la que se sitúa él y a los demás en unan escala de arribas y abajos (pág. 4).

Esta imagen deviene del modelo autoritario e impositivo que acarreaba las atribuciones de control y evaluación de la supervisión/inspectoría educativa. Se traslada a la nueva función de asesoría como una suerte de heredad institucional dentro del sistema educativo. La jerarquización actúa a modo de argumento ad hominen: el accionar de los equipos de asesoría es vertical porque son los asesores educativos quienes lo asumen. La percepción se ratifica con la asesoría externa por la experticia administrativa/pedagógica, manejo de la normativa y formación académica de los asesores ministeriales y zonales. En la asesoría 
educativa interna se confirma pues quienes la cumplen son las autoridades institucionales que tienen los lugares altos en la estratificación organizacional del plantel.

Por el lado de la horizontalidad se tiene a las definiciones establecidas por el Ministerio de Educación (2013) en el MNASGE. En este documento se precisa que "la asesoría educativa se caracteriza por ser un proceso de carácter horizontal, democrático, participativo, respetuoso del saber del docente, centrado en la institución educativa, formativo, profesional y colaborativo." (p. 11). La perspectiva en la transformación de la anterior supervisión es que la asesoría externa no se constituya en una autoridad administrativa de las instituciones educativas. Para ello no se asignó atribuciones de entrega de licencias o permisos, el control de asistencia y puntualidad, la evaluación individual de autoridades o docentes. Esto da cierta igualdad jurídica entre autoridades y los equipos de asesoría educativa y evita actos de coacción o dependencia. La horizontalidad permite la construcción de la alteridad basados en el diálogo entre estos actores educativos. Es "una apertura al otro, que parte de la íntima convicción, en los niveles más profundos de la psique, de que el otro vale, que puede aportarnos algo" (Santos, 2005, p. 6). Este convencimiento es parte del perfil de asesoría y que se ratifica en los momentos de inducción a la gestión y en su capacitación profesional. Por lo demás el otro no es lejano pues las personas que hacen asesoría generalmente su anterior función fue la de ser autoridad institucional.

Las respuestas de los equipos de asesoría consultados consideran que el trabajo horizontal es el que predomina en el ejercicio de su función. En el cuadro 9 se ve que el $73 \%$ está "Totalmente de Acuerdo" y el 17 \% "De Acuerdo" (90 \% acumulado) en esta característica de la función asesora. Solamente el $3 \%$ señala que está "En desacuerdo" y el $7 \%$ «Ni de acuerdo ni en desacuerdo». La relativa igualdad legal y la actitud de apoyo de la mayoría de equipos de asesoría ha contribuido a cimentar este rasgo de horizontalidad. La asesoría es al culmen de la carrera educativa que se inicia como docente y pasa al cumplimiento de distintas responsabilidades incluida la de ser autoridad institucional. Esto genera empatía entendida como capacidad de reconocer el conjunto de gestión de una autoridad o equipo directivo por parte del asesor.

En la función de asesoría educativa prevalece la idea de un trabajo con horizontalidad por sobre el trabajo vertical con las autoridades institucionales.

\begin{tabular}{lc}
\hline Totalmente de acuerdo & $73 \%$ \\
De acuerdo & $17 \%$ \\
Ni de acuerdo ni en desacuerdo & $7 \%$ \\
En desacuerdo & $3 \%$ \\
Totalmente en desacuerdo & $0 \%$ \\
\hline
\end{tabular}

Cuadro 9: Campo de tensión 6: Verticalidad versus Horizontalidad

El séptimo campo de tensión concierne a la permanencia o desaparición de la función de asesoría dentro del sistema educativo ecuatoriano. La desaparición corresponde al limitado número de asesores y asesoras que tienen nombramiento oficial hacia el año 2019 y que son 146 a nivel nacional. Como se señaló en el cuadro 3, este número está alejado de los 2260 proyectados pues estaría cubierto solamente el 6,5\%. Incluso se halla lejos de tener al menos un asesor/asesora por cada uno de los 1134 circuitos educativos existentes. El otro dato que corrobora esta percepción de desaparición de la función es que este número de funcionarios de asesoría externa disminuye ostensiblemente. Ello debido a que en buena parte están más o menos cercanos a la jubilación y sus partidas no han sido repuestas con otros funcionarios. También sienten los equipos de asesoría que su labor no es suficientemente valorada por las autoridades del nivel central. Incluso en alguna de las

Licencia Creative Commons Atribución 4.0 Internacional (CC BY 4.0)

Revista Cátedra, 3(3), pp. 88-110, septiembre-diciembre 2020. e-ISSN: 2631-2875

https://doi.org/10.29166/catedra.v3i3.2406 
administraciones pasadas su función estuvo en entredicho ante el mismo ministro del ramo. Adicionalmente, tienen la percepción de que los demás estamentos del ministerio, zonas y distritos, desconocen el alcance y responsabilidades de la asesoría educativa. Estos datos cuantitativos y de apreciación, contribuyen a la falta de posicionamiento de la asesoría en el conjunto de la autoridad educativa nacional. Es probable que esta situación sea consecuencia de la diversidad de caracterizaciones que enmarca la asesoría educativa. Tamayo y Sandó (2011) señalan varios términos en esta designación: "función, actividad, acciones, servicio, proceso, entre otros, que evidencian diferencias sustanciales de criterios y reflejan incoherencia en su aparato conceptual metodológico" (p. 6). Esta dispersión haría que, en el caso ecuatoriano, la figura del asesor y la función de la asesoría educativa se halle total o parcialmente invisibilizada. El desconocimiento «de lo que hace la asesoría» es un lugar común en los niveles central, zonal y distrital de la autoridad educativa nacional.

La continuidad de la función asesora está asegurada por su importancia dentro del sistema educativo. El principal aspecto que destaca es su ubicación dentro de la estructura. Al ser «actores de frontera», se hallan entre el nivel central y el distrital/circuital. Este es un locus de privilegio: el equipo de funcionarios actúa como mediador entre las disposiciones del nivel central y los requerimientos del nivel local. La capacidad de mediación está validada y ratificada por las experticias administrativas, pedagógicas y de formación académica que es el perfil del asesor/asesora. Este equipo permite que las disposiciones, fundamentalmente las pedagógicas, del nivel central, puedan ser ejecutadas en las instituciones educativas de una manera eficiente y efectiva. Además de las pedagógicas, la orientación que realizan los equipos asesores en las demás dimensiones de los estándares, se caracteriza por su complejidad. Standaert (2012) al respecto menciona que:

el eje del nivel central hacia el nivel local es multidimensional. Algunos aspectos se delegan al nivel local y otros son centralizados, resultando una y otra vez en un equilibrio complejo de aspectos que son centralizados y de otros que se definen a nivel local. La cantidad de aspectos, su importancia y la forma cómo están organizados define el grado de centralización o descentralización de un sistema (p. 60).

La superación de estas tensiones por la vía de la comprensión integral de la gestión institucional en un justo medio dinámico con el nivel central requiere del accionar de un equipo de asesoría. Mantener el enfoque hacia la institución educativa como es la tendencia, con la debida flexibilidad y un equilibrio con las disposiciones, es posible con la asesoría. El reto de la asesoría, como equipo con capacidad de comprender las normas y responsabilidades de los actores educativos, es alcanzar la autonomía pedagógica institucional. Su relevancia consiste en superar estas contradicciones. Con el conocimiento de su importancia de mediación y la relevancia como función, la asesoría tendrá un soporte de estabilidad dentro del sistema.

¿Cómo perciben los asesores y asesoras que respondieron la encuesta, su permanencia dentro del sistema? En el cuadro 10 se puede ver que una tercera parte de asesores piensa que las disposiciones de las autoridades del nivel central no apuntan a la estabilidad de la función en el sistema. El otro tercio de encuestados señala "Ni de acuerdo ni en desacuerdo" que implica incertidumbre. El tercio final percibe que habría estabilidad y que la función de asesoría educativa se mantendrá en la estructura educativa nacional. La mayoría manifiesta el dilema sobre la estabilidad de la función y por ende su propia permanencia laboral. Se puede presumir que esta inseguridad tiene niveles de impacto en la gestión de la asesoría educativa. Los resultados apuntan a que si se quiere lograr eficiencia y eficacia en la gestión

Licencia Creative Commons Atribución 4.0 Internacional (CC BY 4.0)

Revista Cátedra, 3(3), pp. 88-110, septiembre-diciembre 2020. e-ISSN: 2631-2875 https://doi.org/10.29166/catedra.v3i3.2406 
asesora es necesaria la comunicación firme de los decisores políticos en cuanto a su estabilidad. Esta decisión tiene base legal a nivel adjetivo y sustantivo y en la importancia que la función asesora tiene en todo el sistema. El MNASGE considera un tiempo de transición de hasta cinco años para implantar la función (MINEDUC, 2013).

En este período se deberá asimismo avanzar progresivamente en la puesta en marcha de todos los requisitos, lo que significa generar indicadores para un sistema informatizado de monitoreo y diseñar el sistema, desarrollar y acordar estándares de calidad, entrenar a las instituciones educativas en procedimientos de autoevaluación y capacitar a los agentes en las dos funciones que comprende el Modelo: asesoría y auditoría educativa.

Las decisiones de las autoridades estatales hacen pensar que la función de asesoría educativa permanecerá estable antes que desaparecer.

\begin{tabular}{lc}
\hline Totalmente de acuerdo & $20 \%$ \\
De acuerdo & $13 \%$ \\
Ni de acuerdo ni en desacuerdo & $30 \%$ \\
En desacuerdo & $30 \%$ \\
Totalmente en desacuerdo & $7 \%$ \\
\hline
\end{tabular}

Cuadro 20: Campo de tensión 7: Desaparición versus Estabilidad

Estas exigencias han sido satisfechas ya en general. Está definida la importancia de la asesoría dentro del sistema como ente orientador de las instituciones hacia la consecución de los estándares de calidad educativa. Se tienen mecanismos validados de selección de los nuevos asesores y procesos inducción de quienes ganan el derecho laboral a ejercer la función. Es tiempo que quienes ejercen los altos cargos de la autoridad educativa nacional tomen la decisión política de implementar el modelo conforme a la realidad del país.

\section{Conclusiones}

La conclusión principal de este estudio es que el nivel central prevalece sobre los niveles desconcentrados incluido el institucional educativo. Parte de esta prevalencia involucra al nivel de coordinación zonal que para Quito y Guayaquil incumbe a las subsecretarías metropolitanas de educación. El predominio corresponde a la determinación de los procesos y productos que los equipos de asesoría educativa deben ejecutar en las escuelas y colegios.

La segunda conclusión, es que es posible lograr un equilibrio en los campos de tensión. La imposición autoritaria de cualquier tipo de algún nivel sobre otro en materia educativa y pedagógica es inadecuada para el conjunto del sistema. Al ser los equipos de asesoría educativa los principales actores de frontera entre los distintos niveles del sistema, su accionar brinda equidad a la estructura. La asesoría es quien proporciona un balance entre las determinaciones del centro y las realidades de la periferia.

La tercera conclusión es que la función de asesoría es la única instancia que puede hacer mediación de alto nivel técnico entre los niveles del sistema. El espacio de gestión de los equipos de asesoría a nivel de instituciones educativas es diferenciado. Los productos y procesos determinados por los niveles central y zonal son orientados conforme a la realidad institucional propia de cada centro educativo. Los equipos de asesoría educativa tienen la experticia para lograr esta mediación que procura una atención específica a la institución 
escolar. Se determina que una de las características esenciales de la asesoría es su horizontalidad en relación con los equipos directivos institucionales. Esto redunda en su capacidad de intervención balanceada y técnica entre los diferentes niveles del sistema educativo, en especial con los centros escolares.

Como cuarta conclusión se establece que hay necesidad de que las autoridades ministeriales como decisores políticos dentro del sistema aseguren la permanencia de la función de asesoría educativa. Más allá de las coyunturas, independientemente de su paso temporal por el ministerio, las autoridades del nivel central deben afirmar la estabilidad de los funcionarios permanentes. Implica la ampliación paulatina de los equipos de asesoría educativa y la restitución de los que se desvinculan por renuncia o jubilación. El número de asesores educativos puede ser de cuatro por cada distrito para un total nacional de 560 . Habría que considerar el otro actor esencial del MNASGE que es la auditoría educativa. En este caso un mínimo requerido es dos por distrito, es decir se necesitarían 280 auditores educativos. 


\section{Bibliografía}

Aguerrondo, I. y Xifra, S. (2011). La Planificación Estratégica Situacional como método de gobierno. Reorganización de la supervisión educativa en Ecuador. Recuperado el 20 de marzo de 2020 de: http://www.asociacionag.org.ar/pdfcap/6/AGUERRONDO XIFRA.pdf

Aguerrondo, I. y Xifra, S. (2012a). Análisis de una política pública. Reorganización de la supervisión educativa en Ecuador en un contexto de cambio estructural. Revista Análisis Público. Año 1/No 1/junio 2012. Universidad de Valparaíso, Chile. Recuperado el 18 de enero de 2020 de: http://analisispublico.administracionpublicauv.cl/wp-content/uploads/2012/10/03.pdf

Aguerrondo, I. y Xifra, S. (2012b). La supervisión educativa en Ecuador. Del control jerárquico al asesoramiento/monitoreo. Recuperado el 20 de enero de 2020 de: https://studylib.es/docmanager.html?id=8918530\&justuploaded=yes

Aguerrondo, I. (2013). El rol de la supervisión educativa en la gestión de las políticas públicas. Educar 2013, vol. 49/1. IIPE/UNESCO Sede Buenos Aires. Argentina. Recuperado el 20 de enero de 2020 de: https://ddd.uab.cat/pub/educar/educar a2013m16v49n1/educar a2013m1-6v49n1p13.pdf

Antúnez, S. (2012). Principios generales de la asesoría a los centros escolares. En: "La asesoría técnico pedagógica: guía para personal de nuevo ingreso". Antología. Secretaría de Educación de Veracruz. Recuperado el 11 de febrero de 2020 de: https://documentospreescolar.files.wordpress.com/2013/05/antologia-atps.pdf

Casanova, M. (2005). Supervisión, evaluación y calidad educativa. Revista de la Asociación de Inspectores de Educación de España. Núm. 1: Julio (2005). Recuperado el 17 de mayo de 2020 de: https://avances.adide.org/index.php/ase/article/view/2

Carron, G. y De Grauwe, A. (2003). Cuestiones de actualidad en supervisión: una revisión de la literatura. UNESCO - Instituto Internacional de Planeamiento de la Educación.

Cevallos, P. (2016). Cambiar la arquitectura institucional del sistema escolar para mejorar la calidad educativa en Ecuador. En Grupo FARO. "Hacia una Sociedad Educadora: Propuestas para el Debate". MingaLibro No.1. Quito. Recuperado el 13 de julio de 2020 de: https://grupofaro.org/wp-content/uploads/2019/08/MINGALIBROHacia-una-Sociedad-Educadora.pdf

Cortés, M. y León, M. (2004). Generalidades sobre Metodología de la Investigación. Universidad Autónoma del Carmen. México. Recuperado el 8 de agosto de 2020 de: http://www.unacar.mx/contenido/gaceta/ediciones/metodologia investigacion.pdf

Fullan y Hargreaves (1999). La escuela que queremos. Recuperado el 11 de julio de 2020 de http://pdfhumanidades.com/sites/default/files/apuntes/FULLAN\%20Michael\%20 y\%20Hargreaves $\% 20 \% 2$ C $\% 20$ La $\% 20$ Escuela $\% 20$ que $\% 20$ Queremos.pdf 
Gómez, M., Franco, M., Hamui, L. y Cabrera, N. (2017). La educación como campo de tensión y articulación. Un ejercicio de construcción conceptual para pensar la gestión, la evaluación y la interculturalidad en el marco de la reforma educativa de 2013 en México. Congreso Nacional de Investigación Educativa. Recuperado el 6 de agosto de 2020

de: http://www.comie.org.mx/congreso/memoriaelectronica/v14/doc/simposios/135 7.pdf

López, L. (2010). La construcción de la asesoría en el acompañamiento pedagógico. Mexicali, México. Recuperado el 10 de febrero de 2020 de: https://docplayer.es/59226885La-construccion-de-la-asesoria-en-el-acompanamiento-pedagogico.html

Ministerio de Educación y Cultura - MEC. (2010). Modelo Nacional de Supervisión Educativa. Recuperado el 20 de marzo de 2020 de: https://www.educar.ec/noticias/calidad.html

Ministerio de Educación. (2013). Modelo Nacional de Apoyo y Seguimiento a la Gestión Educativa. Recuperado el 12 de febrero de 2020 de: https://educacion.gob.ec/wpcontent/uploads/downloads/2014/03/MNASGE aprobado 18dic.pdf

Ministerio de Educación. (2014). Código de Ética del Asesor Educativo. Dirección Nacional de Asesoría Educativo. Quito.

Ministerio de Educación. (2015). Reglamento General a la Ley Orgánica de Educación Intercultural. En vigencia desde el 19 de julio del 2012. Versión Codificada por la Dirección Nacional de Normativa Jurídico Educativa. Actualizado a 05 de enero del 2015. Recuperado el 3 de mayo de 2020 de: https://educacion.gob.ec/wpcontent/uploads/downloads/2017/02/Reglamento-General-a-la-Ley-OrgAnica-deEducacion-Intercultural.pdf

Ministerio de Educación. (2016). Ley Orgánica de Educación Intercultural. En vigencia desde el 11 de enero de 2011. Versión Codificada por la Dirección Nacional de Normativa Jurídico Educativa. Actualizado a 30 de diciembre de 2016 Recuperado el 3 de mayo de 2020 de: https://educacion.gob.ec/wpcontent/uploads/downloads/2017/05/Ley-Organica-Educacion-InterculturalCodificado.pdf

Ministerio de Educación. (2017a). Estatuto orgánico de gestión organizacional por procesos del Ministerio de Educación. Acuerdo No. 020-12, publicado el 25 de enero del 2012. Recuperado el 21 de mayo de 2020 de: https://educacion.gob.ec/wpcontent/uploads/downloads/2017/11/ACUERD0-020-12-ESTATUTO-MINEDUCcodificado 20-09-2017.pdf

Ministerio de Educación. (2017b). Manual para la implementación y evaluación de los estándares de calidad educativa. Recuperado 15 de enero de 2020 de: https://educacion.gob.ec/wp-content/uploads/downloads/2017/12/Manual-parala-implementacion-de-los-estandares-de-calidad-educativa.pdf 
Miranda, Estela; Lamfri, Nora; González, Silvia y Nicolinil, Mariana. (s.f.). Construcción de la regulación política en educación en la década post-reforma. procesos emergentes y efectos en los sistemas educativos provinciales. Recuperado el 9 de agosto de 2020 de: https://revistas.psi.unc.edu.ar/index.php/Cuadernos/article/viewFile/689/651

Mourshed, M., Chijioke, C. y Barber, M. (2012). Cómo continúan mejorando los sistemas educativos de mayor progreso en el mundo. Programa de Promoción de la Reforma Educativa en América Latina y el Caribe - PREAL. (C) 2010 McKinsey \& Company. Recuperado el 14 de julio de 2020 de: http://200.6.99.248/ bru487cl/files/McK61.pdf

Muñoz, M. y Murillo, J. (2010). Un balance provisional sobre la calidad en educación. eficacia escolar y mejora de la escuela. REICE. Revista Iberoamericana sobre Calidad, Eficacia y Cambio en Educación, vol. 8, núm. 2, 2010. Recuperado el 15 de julio de 2020 de: https://www.redalyc.org/pdf/551/55114080011.pdf

Muñoz, A. (2011). La sociedad de control: una mirada a la educación del siglo XXI desde Foucault. Rev. filos. vol.73. Santiago oct. 2017. http://dx.doi.org/10.4067/S0718$\underline{43602017000100317}$

Organización de Estados Iberoamericanos OEI (s.f.) Breve evolución histórica del sistema educativo. Recuperado el 15 de enero de 2020 de: https://www.oei.es/historico/quipu/ecuador/ecu02.pdf

Pautasso, E. (2009). Genealogía de la Educación Inicial en el Ecuador. Extracto del trabajo de grado: Genealogía de la Educación Inicial en el Ecuador: Periodo histórico 1900-2000. Recuperado

de: https://alteridad.ups.edu.ec/index.php/alteridad/article/view/2.2009.06

Piruch, P. (2005). La supervisión educativa en el sistema de educación intercultural bilingüe del ecuador, como un proceso eficiente y eficaz para mejorar la calidad de la educación. Tesis. Instituto de Altos Estudios Nacionales. Recuperado el 22 de enero de 2020 de: https://core.ac.uk/download/pdf/143427304.pdf

RAE-Real Academia Española. (2019). Diccionario de la lengua española. Recuperado el 10 de junio de 2020 de: https://dle.rae.es/antinomia?m=form

Santos, Marcos. (2005). De la verticalidad a la horizontalidad. Reflexiones para una educación emancipadora. Universidad de Granada. Recuperado el 11 de junio de 2020 de: https://dialnet.unirioja.es/servlet/articulo? codigo $=4027583$

SENPLADES. (2012). Secretaría Nacional de Planificación y Desarrollo. Registro Oficial, Edición Especial N 290 del 28 de mayo de 2012. Recuperado el 4 de mayo de 2020 de:

https://www.planificacion.gob.ec/wpcontent/uploads/downloads/2013/05/REGISTRO-OFICIAL DISTRITOS-YCIRCUITOS.pdf

Standaert, Roger. (2012). Inspectorías Educativas en Europa. Un análisis crítico. ACCO LOVANA /LEUSDEN. Edición: Asociación Flamenca de Cooperación al Desarrollo y Asistencia Técnica. VVOB-Ecuador. Quito. 
Tamayo, A. y Sandó, P. (2011). La Supervisión Educativa: fundamentos epistemológicos de un modelo teórico de su dirección. Ciencias Holguín, vol. XVII, núm. 2, abril-junio, 2011, pp. 1-10. Recuperado el 12 de junio de 2020 de https://www.redalyc.org/pdf/1815/181522301005.pdf

Velásquez, E. (2011). Supervisión educativa como agente de poder y control o como factor de cambio social. Universidad Pedagógica Experimental Libertador. Recuperado el 15 de mayo de 2020 de http://bdigital.ula.ve/storage/pdf/kaleido/v8n16/art05.pdf

\section{Autor}

GUSTAVO VALLEJO-VILLACíS. Obtuvo sus títulos de Profesor de Enseñanza Media, Licenciatura en Ciencias de la Educación, mención pedagogía, y Magíster en Desarrollo de la Inteligencia, en la Universidad Técnica Particular de Loja. Maestría en Equidad, Género y Desarrollo Sostenible, mención Género y Educación, en la Universidad Técnica de Ambato.

Actualmente es asesor educativo jubilado del Ministerio de Educación. 